\title{
Dynamics of Target Recognition by Interstitial Axon Branching along Developing Cortical Axons
}

\author{
Martin Bastmeyer and Dennis D. M. O'Leary \\ Molecular Neurobiology Laboratory, The Salk Institute, La Jolla, California 92037
}

\begin{abstract}
Corticospinal axons innervate their midbrain, hindbrain, and spinal targets by extending collateral branches interstitially along their length. To establish that the axon shaft rather than the axonal growth cone is responsible for target recognition in this system, and to characterize the dynamics of interstitial branch formation, we have studied this process in an in vivo-like setting using slice cultures from neonatal mice containing the entire pathway of corticospinal axons. Corticospinal axons labeled with the dye 1,1'-dioctodecyl-3,3,3',3'tetramethylindocarbocyanine perchlorate (or Dil) were imaged using time-lapse video microscopy of their pathway overlying the basilar pons, their major hindbrain target. The axon shaft millimeters behind the growth cone exhibits several dynamic behaviors, including the de novo formation of varicosities and filopodia-like extensions, and a behavior that we term "pulsation," which is characterized by a variable thickening and thin-
\end{abstract}

ning of short segments of the axon. An individual axon can have multiple sites of branching activity, with many of the branches being transient. These dynamic behaviors occur along the portion of the axon shaft overlying the basilar pons, but not just caudal to it. Once the collaterals extend into the pontine neuropil, they branch further in the neuropil, while the parent axon becomes quiescent. Thus, the branching activity is spatially restricted to specific portions of the axon, as well as temporally restricted to a relatively brief time window. These findings provide definitive evidence that collateral branches form de novo along corticospinal axons and establish that the process of target recognition in this system is a property of the axon shaft rather than the leading growth cone.

Key words: axonal arborization; axon guidance; basilar pons; axon pathfinding; collateral branching; corticospinal axons; target recognition; time-lapse imaging; video microscopy
The formation of a proper functioning nervous system depends on the development of precise connectivity between appropriate sets of neurons. A fundamental issue, therefore, in neurobiolugy is defining the mechanisms by which neurons recognize and innervate their targets. For some time, the growth cone has been considered to be responsible for these crucial functions. Recent advances in axon-tracing methods with fluorescent markers and the development of low-light-level video microscopy have permitted observations of living axons in vivo or in situ over time. Time-lapse video microscopy has been used to study the development of axonal projections in both invertebrates ( $\mathrm{O}^{\prime}$ Connor et al., 1990; Sabry et al., 1991; Myers and Bastiani, 1993) and vertebrates (Harris et al., 1987; O’Rourke and Fraser, 1990; Kaethner and Stuermer, 1992; Sretavan and Reichardt, 1993; Godement et al., 1994; Halloran and Kalil, 1994; O'Rourke et al., 1994). These studies have provided important and interesting insights into the behavior of the axonal growth cone as it navigates toward a target or forms a terminal arbor within a target.

Many populations of neurons in the vertebrate brain innervate multiple, widely separate targets by axon collaterals and, therefore, face a unique problem during development. A prominent example of such a neuronal population is that of layer 5 neurons in the mammalian neocortex. Layer 5 neurons form the major

\footnotetext{
Received Sept. 6, 1995; revised Nov. 17, 1995; accepted Nov. 29, 1995.

This work was supported by National Institutes of Health Grant NS31558 and Deutsche Forschungsgemeinschaft fellowship $\mathrm{Ba}$ 1034/4. We thank Dr. Maryellen Daston for helpful comments on this manuscript.

Correspondence should be addressed to Dennis D. M. O'Leary, MNL-O, The Salk Institute, 10010 North Torrey Pines Road, La Jolla, CA 92037.

Dr. Bastmeyer's present address: University of Konstanz, Faculty for Biology, Postfach 5560, D-78434 Konstanz, Germany.

Copyright (C) 1996 Society for Neuroscience $0270-6474 / 96 / 161450-10 \$ 05.00 / 0$
}

output projection of the cortex and establish connections with several targets in the midbrain, hindbrain, and spinal cord. During development, layer 5 axons extend out of the cortex along a spinally directed pathway, and their growth cones seemingly ignore their targets as they grow past them and continue to elongate through the corticospinal tract. The brainstem and spinal targets are later contacted exclusively by axon collaterals extended by corticospinal axons. Evidence obtained from the examination of fixed tissue sections suggests that these collaterals develop by a delayed interstitial branching from the axon shaft (O'Leary and Terashima, 1988; O'Leary et al., 1990; Kuang and Kalil, 1994).

These observations suggest that in this projection system the process of target recognition does not involve the growth cone but, instead, is a property of the axon shaft. Until recently, however, the axon shaft has been considered to be a nonresponsive, quiescent structure with a stable cytoskeleton. For this reason, and because of difficulties in establishing an appropriate experimental system, little attention has been given to characterizing the role of the axon shaft in target recognition and innervation. The projection from cortical layer 5 neurons to the basilar pons is a model well suited for studying this mechanism of target recognition. The basilar pons is innervated by collateral branches that form postnatally along the shaft of corticospinal axons (O'Leary and Terashima, 1988). Here we have used time-lapse video microscopy of fluorescently labeled corticospinal axons to monitor the dynamics of interstitial axon branching and to establish whether these branches form de novo or from remnants of the growth cone.

To study interstitial axon branching in an in vivo-like situation, we developed a slice culture preparation from early postnatal mice in which corticospinal axons could be visualized in the part 
of their tract overlying the basilar pons, as well as in the part just caudal to the pons. We find that the axon shaft within the tract overlying the basilar pons is highly dynamic with multiple sites of activity and that collateral branches form de novo. These axonal behaviors are both spatially and temporally restricted. Our observations demonstrate that the axon shaft millimeters behind the growth cone is actively involved in the process of target recognition.

\section{MATERIALS AND METHODS}

Animals. Mouse pups were obtained from timed-pregnant ICR mice purchased from a local supplier (Harlan Sprague-Dawley, Indianapolis, IN). Pups were born on the 18th or 19th day of gestation; only those born on the 18th day were used in this study. The first $24 \mathrm{hr}$ after birth was designated postnatal day $0(\mathbf{P} 0)$. Pups were anesthetized by hypothermia before all procedures.

Dye application. A $25 \%$ solution of the lipophilic carbocyanine dye 1,1'-dioctodecyl-3,3,3',3'-tetramethylindocarbocyanine perchlorate (DiI; Molecular Probes, Eugene, OR) (Honig and Hume, 1989) in dimethylformamide (Sigma, St. Louis, MO) was used for axonal labeling. Two to four hours after birth, the pups were anesthetized and a small hole was made in the scalp and skull overlying motor or visual cortex. A small amount of the DiI solution was pressure-injected into one cortical hemisphere through a glass micropipette using a picospritzer II (General Valve, Fairfield, NJ). After the pups recovered from anesthesia, they were returned to their mothers.

Histology. To establish the time course of the development of the corticopontine projection in mice, a series of pups was reanesthetized and perfused between late $\mathrm{P} 0$ and $\mathrm{P} 3$ with $4 \%$-formaldehyde in phosphate buffer, $\mathrm{pH}$ 7.4. Serial sagittal sections of the brain and cervical spinal cord were cut at $100 \mu \mathrm{m}$ on a Vibratome. Sections were mounted on glass slides in phosphate buffer and examined and photographed on an upright fluorescence microscope (Nikon Microphot, Tokyo, Japan).

Slice preparation. To prepare tissue slices for video microscopy, pups were reanesthetized $14-20 \mathrm{hr}$ (latc P0) or 38-44 hr (late P1) after DiI injection, and their brains with the cervical spinal cord attached were rapidly dissected in $4^{\circ} \mathrm{C}$ Leibowitz medium supplemented with $0.6 \%$ sucrose (L-15). This tissue was placed dorsal surface down on a nitrocellulose filter (Sartorius, Edgewood, NY), with the hindbrain slightly bent laterally toward the side of the DiI injection (see Fig. 2A). The tissue was embedded in $3 \%$ agar (low gelling temperature agarose; FMC Bioproducts, Rockland, ME). Parasagittal slices $(\sim 1.5 \mathrm{~mm}$ in thickness) were cut using a McIlwain tissue chopper (Brinkman, Westbury, NY) such that the corticospinal tract overlying the basilar pons was exposed. The slice was placed onto the membrane of a Millicell insert (Millipore, Bedford, MA) in a tissue culture dish and covered with medium (L-15 supplemented with $30 \mathrm{~mm} \mathrm{NaHCO}, 0.6 \%$ sucrose, $10 \%$ fetal calf serum, and $5 \%$ rat serum). The medium level was adjusted to keep the surface of the slice barely submerged. The slice was then covered with a transparent membrane (BioFoil 25, Heraeus, S. Plainfield, NJ) that was floated on the thin film of medium covering the slice (see Fig. $2 C$ ). This technique was found to enhance slice survival and yielded viable axons for up to $10 \mathrm{hr}$.

Time-lapse video microscopy. Slices were imaged immediately after preparation. During imaging the slices were kept in an incubation box mounted on an upright microscope (Nikon Microphot FX). The environment in the box was maintained at $37^{\circ} \mathrm{C}$ in $95 \% \mathrm{O}_{2} / 5 \% \mathrm{CO}_{2}$. In addition, a $95 \% \mathrm{O}_{2} / 5 \% \mathrm{CO}_{2}$ mixture was applied directly to the culture dish. The microscope was equipped with a $100 \mathrm{~W}$ mercury epifluorescence light source, a rhodamine filter set, and $10 \times, 20 \times$, and $40 \times$ long working distance objectives. To avoid photodamage to fluorescently labeled axons, video imaging was performed under low-light-level conditions. Neutraldensity filters were placed in the light path to reduce the intensity of the fluorescent light. To reduce the duration of light cxposurc to the axons, an electronic shutter (Uniblitz, Rochester, NY) was placed into the light path. The shutter was controlled by Image-1 software (Universal Imaging, West Chester, PA) to open for $\sim 200 \mathrm{msec}$. Images were taken with a silicon-intensified target (SIT) camera (Hamamatsu, Bridgewater, NJ), averaged over 4 frames, collected on a hard disk, and later transferred to a rewritable digital optical disk recorder. Images shown in Figures 3-7 were transferred to a Macintosh computer and processed with Adobe Photoshop software. After adjusting the contrast and brightness to make all of the panels uniform, the images shown in Figures 3 and 5-7 were "embossed." To emboss an image, the software duplicates the image, shifts the duplicates by 2 pixels, and subtracts them from each other. The shifting angle was chosen for each figure to prevent any loss of axonal or branch detail. This procedure provides an even background and a Nomarski-like appearance of axonal structures.

To select slices for video imaging, the corticospinal tract rostral and caudal to the basilar pons was inspected with the $20 \times$ objective. Only slices with the entire corticospinal pathway intact from the cortex to the cervical spinal cord were used. The slice cultures were made in such a way that the axon tract over the basilar pons and immediately caudal to it could be clearly visualized (see Fig. 2). This is the only portion of the pathway at which axons might be cut during slice preparation. Cut axons could be readily identified. Slices that contained cut axons, or beaded axons, indicating degeneration, were discarded. We also selected slices with a low number (5-50) of labeled axons for video observation, which provided better resolution of individual labeled axons. Most of the axons described in this study were $\sim 20-100 \mu \mathrm{m}$ below the surface, because the resolution decreased in deeper focal planes; axons on the surface of the slice were not monitored.

To avoid photodamage, images were usually taken every $10 \mathrm{~min}$. The focus was continually monitored to ensure that changes in morphology were not caused by changes in focal plane. For another sel of experiments, images were taken every 2 min at slightly higher light levels to increase both image quality and time resolution. These axons were imaged for no more than 1-2 hr. The images presented in Figures 3-5 and 7 were taken with the $40 \times$ objective (Nikon Mplan 40 ELWD, 0.5 numerical aperture), and those in Figure 6 were taken with the $20 \times$ objective (Nikon M Plan 20 SLWD, 0.35 numerical aperture). At the end of each imaging session, a low-power ( $10 \times$ objective) bright-field and fluorescence image was taken to record the relative position of the time-lapsed axon to the basilar pons.

\section{RESULTS}

In initial experiments, we established the time course of development of the corticopontine projection in mice, because previous studies were done in rats. The present studies were done in mice because their brains are much smaller and, thus, it was easier to obtain viable slices containing the entire corticospinal pathway. Corticospinal axons labeled by DiI injections into motor cortex (Fig. $1 A$ ) reach the pyramidal decussation at late $\mathrm{P} 0 /$ early $\mathrm{P} 1$. The main front of these axons is found in the decussation (Fig. 1B). However, the outgrowth is staggered because some growth cones are observed between the pons and the decussation, and others have extended just beyond the pyramidal decussation and into the dorsal funiculus of the cervical spinal cord. At this stage, only a few axons have formed short collateral branches on segments overlying the caudal part of the basilar pons. One day later (P2), the mature pattern, characterized by long collaterals into both the rostral and caudal parts of the basilar pons, is well established (not shown). Axons from the visual cortex reach the basilar pons at late P0. These axons also grow caudally past the pons and begin to extend collateral branches into the rostral part of the basilar pons late on P1. Therefore, as in the rat, the corticopontine projection in mice develops by collateral extension from the axon several millimeters behind the growth cone.

Most of our video imaging was done on slices prepared from late $\mathrm{P} 0$ mice, a time when corticospinal axons from motor cortex begin to form collateral branches in vivo. The layout of the corticospinal pathway and the design of the slice preparation are illustrated in Figure 2. These slices had to fulfill the following criteria: (1) they must contain the entire pathway of layer 5 axons from the cortex to the contralateral spinal cord without injuring corticospinal axons; (2) the corticospinal axon pathway overlying the basilar pons should be near the surface of the slice to allow axonal imaging; and (3) the slice and the labeled axons must be viable for many hours. We have concentrated our time-lapse video microscopy on the portion of the axon shaft within the pathway overlying the basilar pons and just caudal to it, a region that in a 

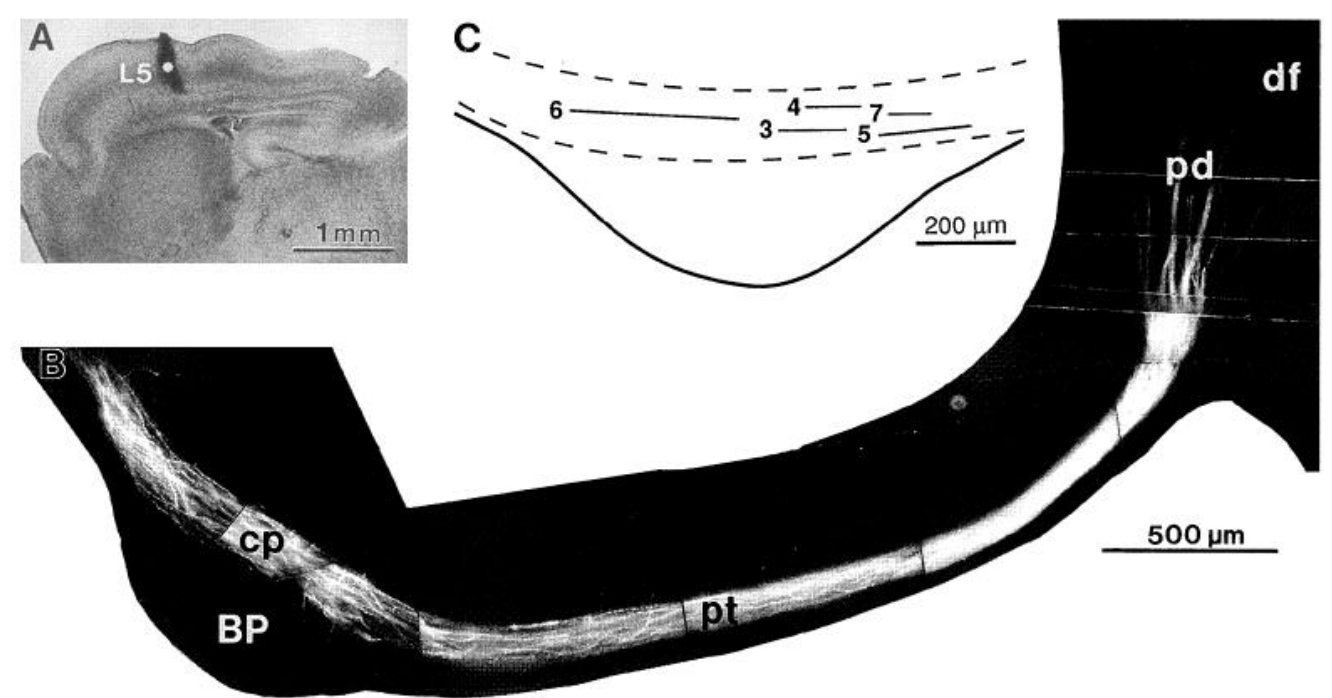

Figure 1. $A, B$, Axons in a late P0 mouse labeled with DiI injected into motor cortex $14 \mathrm{hr}$ earlier. A, Dil injection site (asterisk) involving layer 5 (L5) illustrated in a bright-field image of a sagittal section. $B$, Fluorescence image of a sagittal section of the brainstem. The labeled corticospinal axons pass over the basilar pons $(B P)$ as they extend through the cerebral peduncle $(c p)$ and the pyramidal tract $(p t)$ toward the spinal cord. The main front of labeled axons is in the pyramidal decussation $(p d)$. However, axonal growth is staggered and some growth cones have already entered the dorsal funiculus $(d f)$ of the spinal cord. At this stage, only a few axons have formed short collateral branches at locations in the tract overlying the caudal part of the basilar pons. $C$, Schematic drawing illustrating the location of the axonal segments imaged in Figures 3-7. These axon segments were video-imaged in separate cases in which a much smaller number of axons was labeled.

neonatal mouse is 7-8 $\mathrm{mm}$ distal to the layer 5 cell body and 3-4 $\mathrm{mm}$ behind the growth cone. Most of the corticospinal tract from cortex to the spinal cord was deep in the slice, but the part of the tract overlying the basilar pons, as well as the part just caudal to the pons, was near the medial surface of the slice. We examined $\sim 430$ slices, of which 86 were video-imaged (see Materials and Methods for selection criteria) with images taken at intervals ranging from 1 to $10 \mathrm{~min}$ depending on the case. For video imaging, the DiI injections were much smaller than those used in Figure 1 and labeled between 5 and 50 corticospinal axons. Video imaging of the slices clearly resolved individual corticospinal axons and branches along their length overlying the basilar pons (Fig. 3). The labeled axons were not fasciculated with each other but were distributed over the dorsal-ventral extent of the tract and, therefore, intermingled with corticospinal axons not labeled by the injection. All of the labeled axons were in place at the time the imaging was initiated; no growth cones were observed in the tract overlying the basilar pons during the imaging sessions. All labeled axons could be followed through the tract well beyond the basilar pons; none turned and ended in the basilar pons. To control for artifactual appearance or disappearance of branches, the focus was controlled manually and several focal planes were examined at each time point.

Time-lapse video imaging of the slices revealed that branches can exhibit cycles of retraction and elongation (Fig. $3 A-E$ ) and can form de novo along the axon (Fig. $3 F$ ). Further, we find that the axon segment overlying the basilar pons is highly dynamic, showing several distinct forms of activity along its length. As illustrated in Figure 3, varicosities can appear de novo and disappear, and some move along the axon in both anterograde and retrograde directions. The varicosities appeared to be true swellings of the axon rather than bright inclusions within the axon. We also observed another form of axonal activity characterized by a variable thickening and thinning of short segments of the axon shaft (Fig. 4). This form of axonal activity is clearly visible when consecutive images are played in rapid succession and gives a visual impression that the axon shaft is "pulsating." Axonal pulsation typically lasted for several minutes and could come and go repeatedly at the same location along an axon over hours (Fig. 4). Finally, filopodia-like extensions $2-5 \mu \mathrm{m}$ in length were observed to form de novo. These extensions showed repeated cycles of elongation and retraction, and they even changed their direction (Fig. 4). Although these structures were dynamic, they often persisted to the end of the imaging session.

Some of the filopodia-like extensions eventually grew longer and formed a branch. Further, the de novo extension of filopodialike structures and branches were often associated with a varicosity. Varicosities associated with such axonal extensions tended to remain in place and did not move along the axon. An example of an axon segment overlying the caudal third of the basilar pons is illustrated in Figure 5. A small varicosity formed de novo on this axon (Fig. $5 C$ ) and $\sim 30 \mathrm{~min}$ later a filopodia-like structure emerged from the varicosity (Fig. $5 D$ ). This filopodia-like extension showed repeated cycles of retraction and elongation over the next hour (Fig. 5E,H) until it finally elongated and formed a branch $20 \mu \mathrm{m}$ length (Fig. $5 I, K$ ). This particular branch did not form at a right angle to the axon shaft, as was more typically observed. However, because it formed at the caudal border of the basilar pons (Fig. 1C), it was directed toward the pons. During the imaging session, a second branch formed rostrally along a segment of the axon directly over the pons. This branch was directed in a right angle toward the underlying basilar pons (Fig. $5 A, L$ ).

A single corticospinal axon often had several simultaneously active sites characterized by the extension of filopodia-like structures or the formation of short branches, as illustrated in Figure 6 with an axon labeled by DiI injected in visual cortex. This axon segment spanned one-half of the rostral-caudal extent of the tract overlying the basilar pons (Fig. $1 C$ ) and stayed in focus for an 
A

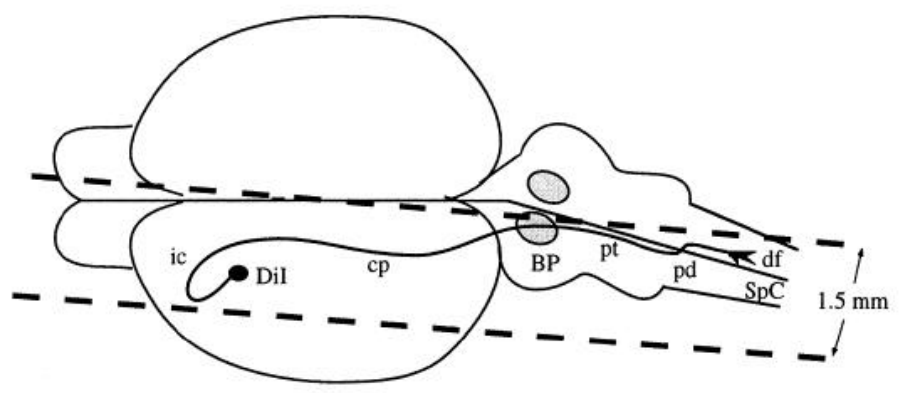

B

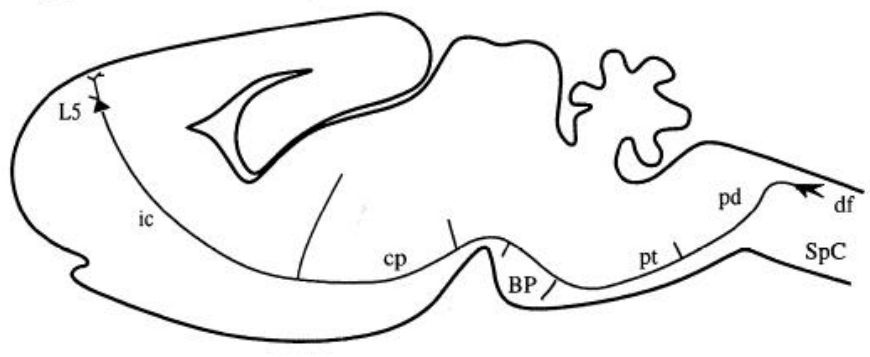

C

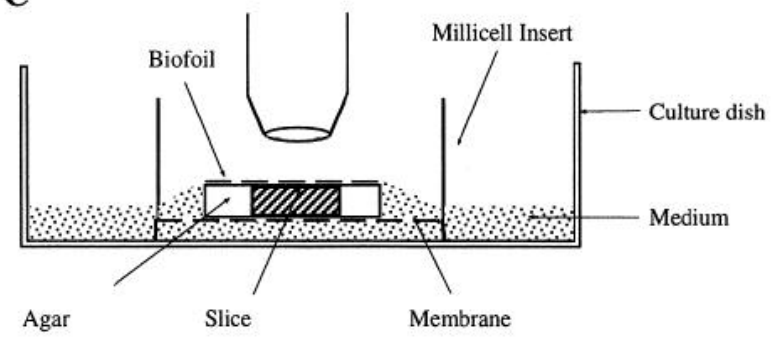

Figure 2. Schematic drawings of the corticospinal tract in newborn mice and the slice preparation used in this study. Dorsal $(A)$ and parasagittal $(B)$ views of the corticospinal tract in newborn mice. DiI-labeled Layer 5 (LS) axons leave the cortex via the internal capsule (ic) and extend through the cerebral peduncle $(c p)$ and the pyramidal tract $(p t)$ into the spinal cord $(S p C)$. At late $\mathrm{P} 0$, the growth cones of these axons have reached the pyramidal decussation $(p d)$ or the rostral part of the dorsal funiculus $(d f)$. At that age, the first axons from motor cortex start to form collateral branches in the tract overlaying the basilar pons $(B P)$. The dotted lines in $A$ indicate the section planes to cut a $1.5 \mathrm{~mm}$ sagittal slice containing the entire corticospinal projection and exposing the pyramidal tract in a region overlaying the basilar pons. This slice preparation is cultured medial face up (the face with the exposed axon tract) in a Millicell Insert $(C)$ as described in Materials and Methods.

extended period of time. When the imaging session began, this axon had already formed a branch overlying the rostral part of the pons (Fig. $6 \mathrm{~A}$ ). In addition, it had at least three more active sites where filopodia-like extensions formed de novo over the next $2 \mathrm{hr}$ (Figs. $6 B, D, E$ ). Two of the filopodia-like structures were extended in the direction of the basilar pons, whereas one pointed dorsally. The filopodia-like extensions were often transient. In this example, the extension marked in Figure $6 B$ disappears in Figure $6 D$ and reappears in Figure $6 E$, whereas the one marked in Figure $6 D$ disappears in Figure $6 E$.

A quantification of this dynamic behavior of the axon shaft is presented in Table 1. Corticospinal axons were labeled by DiI
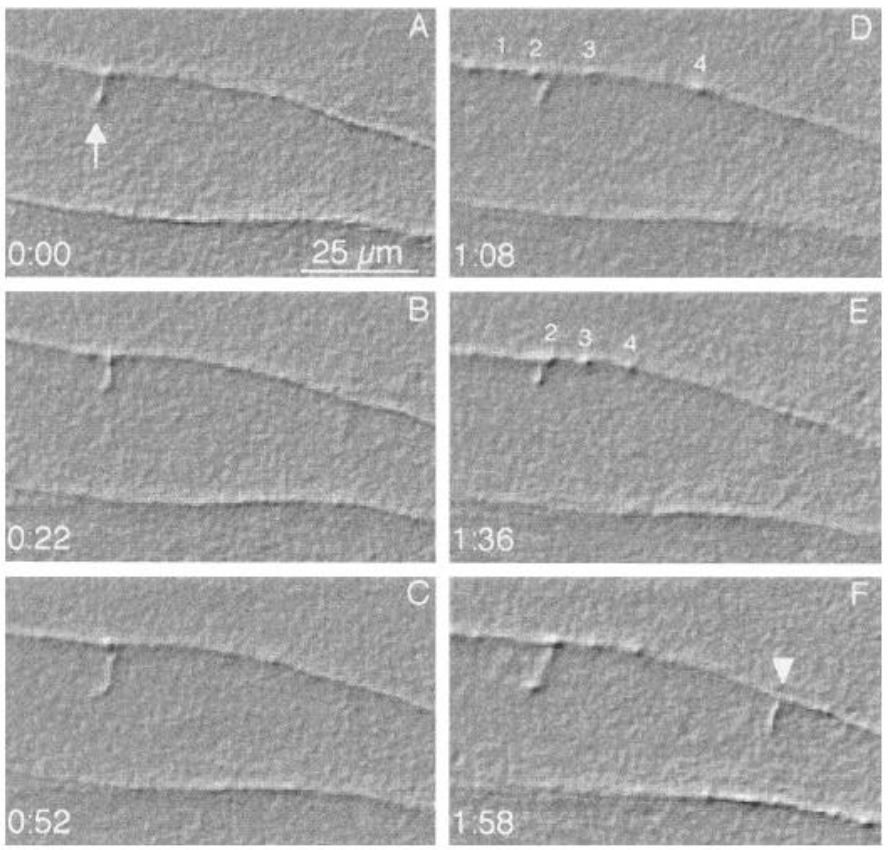

Figure 3. Collateral branches can form de novo along corticospinal axons. Two corticospinal axons labeled with DiI injected into motor cortex and imaged at late P0. One branch (arrow in $A$ ) had already formed at the beginning of the video session. This branch elongated during the first hour $(A-C)$, partially retracted $(D-E)$, and elongated again $(F)$. In addition, a new branch formed de novo on the same axon (arrowhead in $F$ ). Varicosities also form de novo $(1,2,3)$. The varicosities move along the axons in both anterograde $(2$ in $D, E)$ and retrograde $(4$ in $D, E)$ directions. Sagittal section; dorsal is to the top, rostral to the left. Time is given in $\mathrm{hr}$ and min. These images were embossed (see Materials and Methods).

injected in the motor cortex early on $\mathrm{P} 0$, and slices were prepared late on P0. Axonal segments overlying the caudal third of the basilar pons were time-lapse video-imaged at 5-10 min intervals. We counted branches (defined as $5 \mu \mathrm{m}$ or longer) observed to form de novo along all axons for which at least a $200 \mu \mathrm{m}$ segment was in focus for more than $2 \mathrm{hr}$. Fifteen axons met these criteria and were analyzed from images collected over a total of $64 \mathrm{hr}$ (mean $257 \mathrm{~min} / \mathrm{axon}$ ). Thirteen of the fifteen axons (86\%) were observed to form at least one branch during the imaging sessions, suggesting that essentially all corticospinal axons originating from motor cortex are competent to form a branch. The mean frequency of branch formation was $0.15 \pm 0.03$ branches $\cdot 100$ $\mu \mathrm{m}^{-1} \cdot \mathrm{hr}^{-1}$. Branch extension was directed, with $77 \%$ of these branches extending ventrally from the parent axon toward the basilar pons. About $55 \%$ of the branches observed to form de novo persisted for at least $1 \mathrm{hr}$ and were still evident at the end of the imaging session. If all of these branches were permanent (which is not expected), these figures indicate that $\sim 1.6$ stable branches would form along a $100 \mu \mathrm{m}$ length of axon over a $24 \mathrm{hr}$ period. Even given the impreciseness of some of these variables, this predicted number of branches corresponds well with our findings from fixed brain sections-that most corticospinal axons extend branches into the pontine neuropil, and some axons even have two or three pontine branches (O'Leary and Stanfield, 1985; O'Leary and Terashima, 1988) (M. Bastmeyer, M. Daston, and D. O'Leary, unpublished observations).

In other slices prepared in the same manner at the same age, we imaged over time seven corticospinal axons just caudal to the 

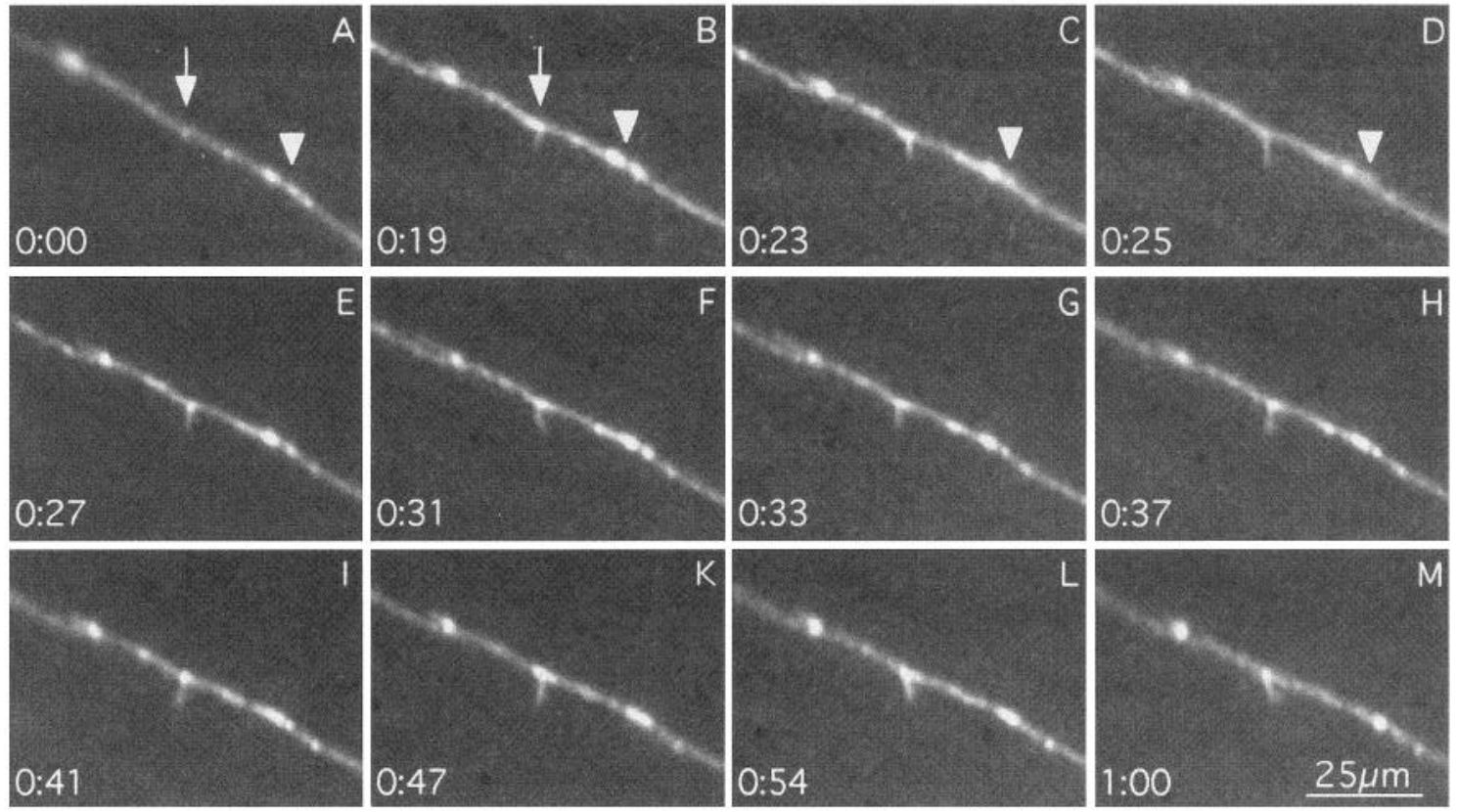

Figure 4. The axon segment overlying the basilar pons is highly dynamic. Corticospinal axon labeled with DiI injected into motor cortex and imaged at late P0. A filopodia-like extension forms (arrow in $A, B$ ) and shows repeated cycles of elongation and retraction as well as slight changes in direction $(C-M)$. The axon shaft pulsates, characterized by thickening or thinning over lengthy stretches (arrowheads in $A-D$ ). Sagittal section; dorsal is to the top, rostral to the left. Time is given in hr and min. These images show the actual fluorescence appearance without embossing.

basilar pons (200 $\mu \mathrm{m}$ segment/axon), a region of the corticospinal tract where branches are not observed in vivo. In contrast to the activity observed along the segments of these axons overlying the basilar pons, we observed no branch formation, filopodia-like activity, or pulsation along the axon segments in the tract just caudal to the pons over a total imaging time of $10.5 \mathrm{hr}$ (mean 90 min/axon). The difference in branching activity overlying the pons $\left(0.46 \pm 0.09\right.$ branches $\left.\cdot 200 \mu \mathrm{m}^{-1} \cdot 90 \mathrm{~min}^{-1}\right)$ versus just caudal to the pons $\left(0.0 \pm 0.0\right.$ branches $\left.\cdot 200 \mu \mathrm{m}^{-1} \cdot 90 \mathrm{~min}^{-1}\right)$ is statistically significant (Student's unpaired $t$ test, $p=0.0024$ ). Therefore, activity is differentially regulated along the axon shaft.

Because of limits on the time that the brain slices are viable, we were not able to time-lapse video image the entire sequence of collateral formation uninterrupted from the initiation of a branch in the axon tract through its extension into the pontine neuropil. Thus, in a separate set of experiments, we again labeled corticospinal axons by DiI injected in motor cortex early on P0 but did not prepare slices until late $\mathrm{P} 1$. By this age, long branches have already formed and extended into the basilar pons (Fig. 7). Because all of the labeled growth cones have past the pons by the ages at which we did the video imaging, these long collaterals must have formed by the de novo extension of branches along the shaft of corticospinal axons as observed at the earlier imaging time point, rather than from growth cone bifurcation or from a remnant of the growth left behind on the axon shaft. Time-lapse video imaging of 14 axons ( $200 \mu \mathrm{m}$ segment/axon) for a total of $\sim 60 \mathrm{hr}$ (mean $264 \mathrm{~min} / \mathrm{axon}$ ) did not reveal any branch formation or filopodia-like activity along these axons. Over this same period, however, the branches themselves showed considerable activity within the basilar pons. This activity was characterized by the lengthening and shortening of secondary branches as well as the elaboration of complex arbors within the pons (Fig. $7 A-C$ ). This activity was restricted to the pontine neuropil; the branches showed no activity along their segments that traversed the axon tract from the parent axon to the basilar pons. Therefore, in addition to a spatial restriction, activity along the axon shaft is temporally restricted.

Our findings indicate that branches form de novo along the length of corticospinal axons, and that these branches extend across the axon tract and into the basilar pons. Nevertheless, we have never observed a branch tipped with a growth cone-like ending. Instead, the branches, as well as the shorter filopodia-like extensions from the parent axons, have either a blunt or a tapered ending (Figs. 3-6). Secondary branches that form within the pontine neuropil also lacked growth cone-like endings (Fig. 7).

\section{DISCUSSION}

To investigate the dynamics of target recognition by developing corticospinal axons, we have used time-lapse video microscopy to study in living brain slices the formation of collateral branches to the basilar pons, a major hindbrain target of these axons. We show that the axon shaft within the pathway overlying the basilar pons is highly dynamic and exhibits several types of activity, including the de novo formation of varicosities, filopodial-like extensions and branches, as well as axonal pulsation. These types of axonal activities are both spatially and temporally restricted. These observations indicate that the axon shaft itself, several millimeters behind the growth cone, is actively involved in the process of target recognition.

Because our study was done in living brain slices rather than in living animals it is necessary to be certain that the types of activity that we observe along the axon shaft are normal axonal behavior and not induced by the experimental procedures. Main sources of potential damage are injury of the axons during the slice preparation or light-induced photodamage to fluorescently labeled axons. However, we find that axons that were cut during the slice preparation or that were intentionally overexposed to fluorescent light show a different behavior: within minutes they have a beaded 

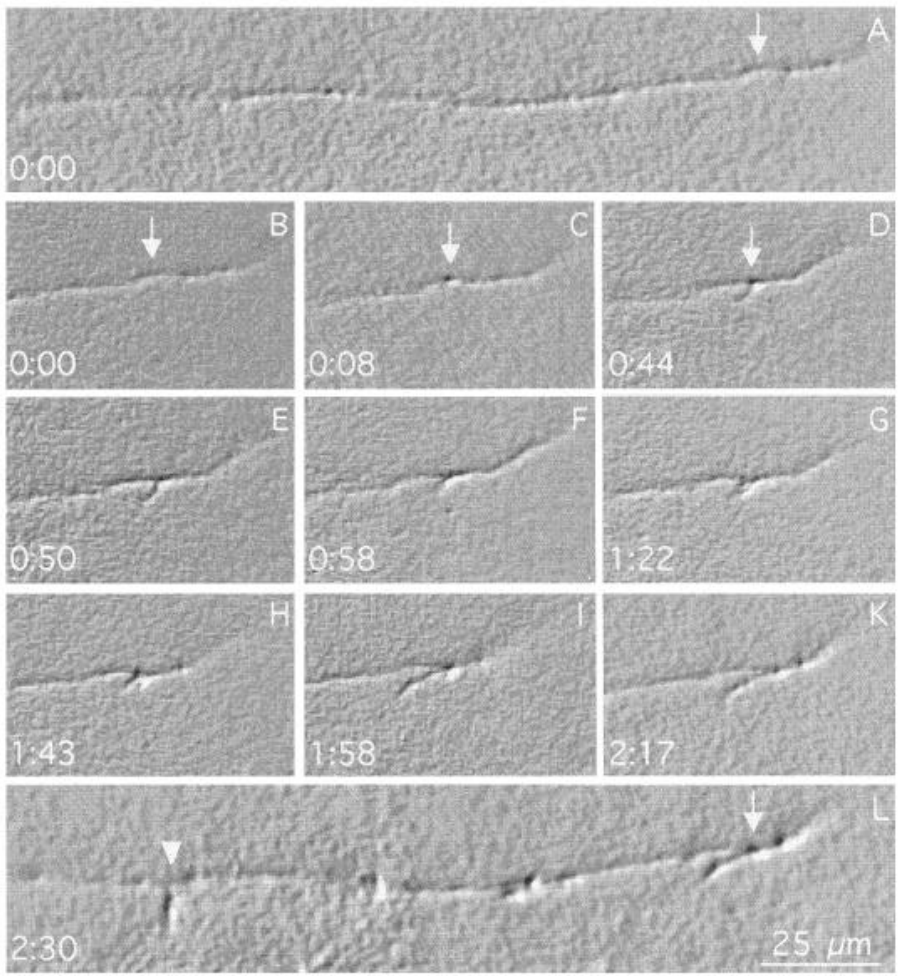

Figure 5. Collateral branch formation is often associated with an axonal varicosity. Corticospinal axon labeled with DiI injected into motor cortex and imaged at late P0. A collateral branch formed de novo at the site marked with an arrow in $A$ (compare with $L$ ). The site marked with an arrow overlying the caudal third of the basilar pons $(B-K)$ shows the sequence in branch formation. A small varicosity formed de novo at this site (arrow in $B, C$ ), and 30 min later a filopodia-like structure emerges at the varicosity (arrow in $D$ ). This filopodia-like extension showed repeated cycles of retraction and elongation over the next hour $(E-K)$. This branch did not form at a right angle to the axon shaft, as is more typically observed. However, because it formed at the caudal border of the basilar pons (Fig. $1 C$ ), it is directed toward the pons. Another branch that formed during the imaging session (arrowhead) extended from a more rostral segment of the axon directly over the pons and is directed in a right angle toward the underlying basilar pons (arrowhead in L). Sagittal section; dorsal is to the top, rostral to the left. Time is given in hr and min. These images were embossed (see Materials and Methods).

appearance and finally break up into several bright fluorescent spots. In addition, because the specific "target-recognition" phenomena that we observe occurred in the tract overlying the basilar pons and not caudal to it, and only in slices prepared at the proper ages, we can rule out that they are simply induced by poor culture conditions or gradual photodamage.

Several pieces of evidence argue that our observations are truly normal behaviors of the axon shaft and are not attributable to a late-arriving growth cone tracking along a preexisting axon. First, corticospinal axons labeled from one site in the cortex are widely distributed over the depth of the tract; because we have restricted our time-lapse observations to cases in which few axons are labeled, it is unlikely that each of the axons that we have studied has later-growing labeled axons tracking along them in a tightly fasciculated manner such that they are undetectable. Second, the activity that we observe occurs repeatedly at specific points along the axon shaft; in contrast, activity attributable to a tracking growth cone would gradually progress caudally down the axon as that growth cone continues its spinally directed growth. Third, the activity is prominent along the axon segment in the tract overlying the basilar pons but not along the axon segment in the tract just caudal to the pons.

Motility in the axonal growth cone is mainly based on dynamic changes in the microtubular and actin cytoskeleton. However, the activity of these proteins is usually shut down in the axon shaft, which has been typically viewed as a quiescent structure (for review, see Mitchison and Kirschner, 1988). Elongating branches imaged in our slice cultures, as well as branches labeled in fixed tissue sections (O'Leary and Terashima, 1988), are not tipped with morphologically obvious growth cones but, instead, resemble large filopodia of advancing growth cones. Nonetheless, branch formation and extension necessarily require the activation of motile machinery initially at a localized region along the axon shaft and later in the tip of the extending branch.

Clearly, the de novo formation of collateral branches along an axon shaft several millimeters behind its growth cone requires substantial changes in the cytoskeleton. Observations of spontaneous axon branching of hippocampal neurons in dissociated cell culture suggest that branch formation is associated with a local fragmentation of microtubules (Yu et al., 1994). Interestingly, a fragmentation or depolymerization of microtubules is directly related to changes in the actin cytoskeleton and, therefore, to an increase in the surface activity of an axon (Bray et al., 1978; Joshi et al., 1986). When sensory axons grown in culture were treated with colchicine to depolymerize microtubules, they formed lamellipodia-like structures along their length (Bray et al., 1978), a phenomenon that may be a correlate of the pulsation that we observed on corticospinal axons. Although the signal transduction pathway that leads to cytoskeletal changes in these developing axons is unknown, it is likely that the cues that induce collateral branching act via such a signaling cascade to promote a localized fragmentation or depolymerization of microtubules.

The point of origin of an axon collateral is typically associated with a varicosity (O'Leary and Terashima, 1988; Nakamura and O'Leary, 1989; Ghosh and Shatz, 1990; Bhide and Frost, 1991; Kadhim et al., 1993; Halloran and Kalil, 1994). We find that de novo branch formation is often associated with axonal varicosities. In these instances, the varicosity itself forms de novo and becomes a site of subsequent extension of filopodial-like structures. A proportion of the filopodial-like structures is transient, but many of them elongate into longer branches and are maintained at least until the end of the recording session. An ultrastructural study of the developing corticospinal tract at ages before branches are evident has described varicosities along corticospinal axons (de Kort et al., 1985). Most of these varicosities are characterized by a light cytoplasm; however, some contain a dark cytoplasm and closely resemble the cytoplasm of growth cones. Thus, the varicosities related to branching could be regions of local tubulin and actin depolymerization and may provide a pool for the proteins that are necessary for the formation of a collateral branch.

Our observations suggest a sequence in the formation of a collateral branch: first is the induction of an active site on the axon shaft, often overtly typified by a varicosity; second is the directional extension of a filopodia-like process; and third is the further elongation of this process and its stabilization as a branch. Whereas the actin cytoskeleton is likely a prominent actor in the first two steps, branch stabilization might occur by microtubules entering the branch. This scenario is suggested by the findings that growth cone advance is mediated by actin filaments, whereas growth cone turning is accompanied by the extension of microtubules into the filopodia that lead the turn (Sabry et al., 1991). 

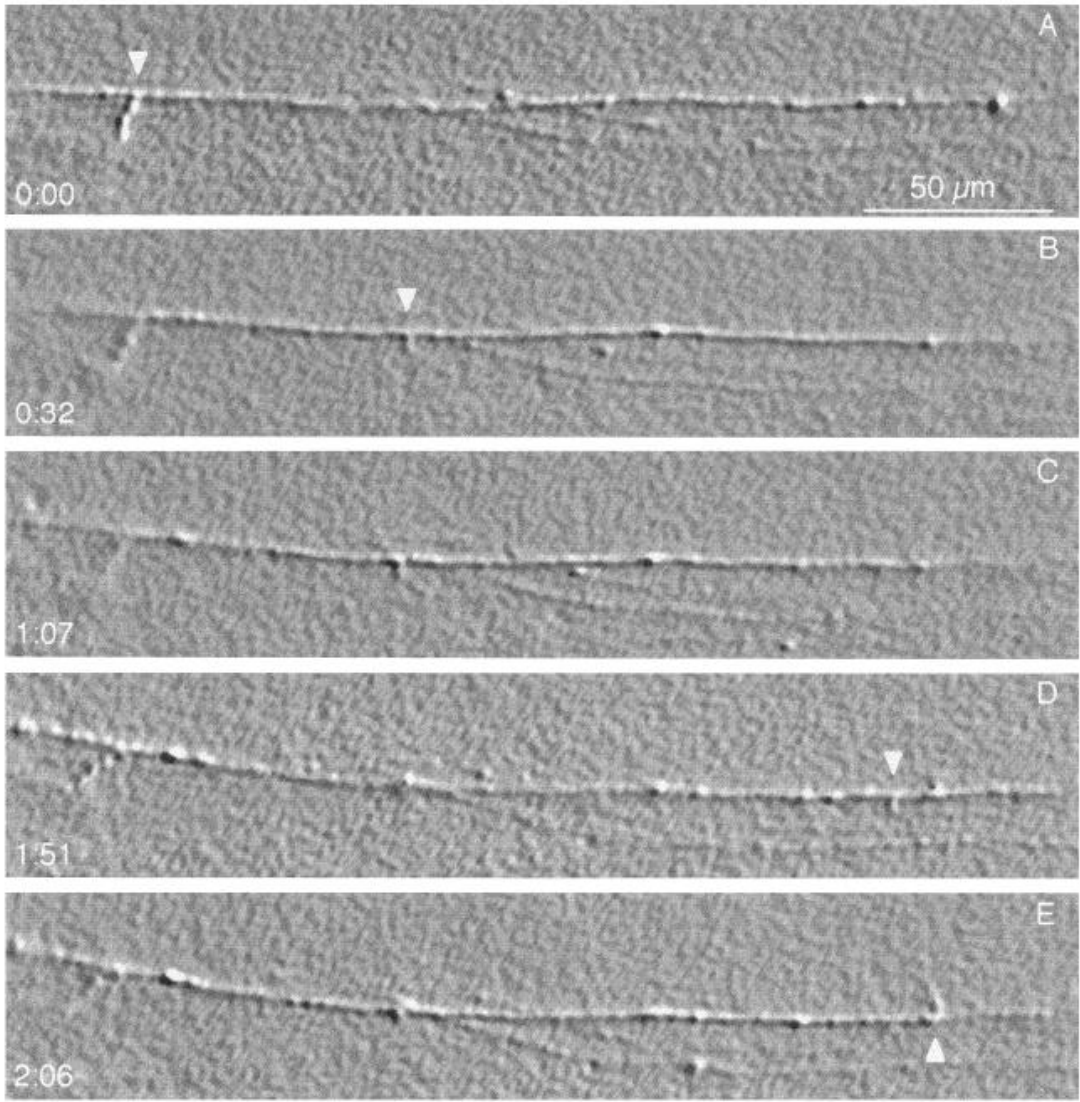

Figure 6. An individual corticospinal axon can have several active sides. Corticospinal axon labeled with DiI injected into visual cortex and imaged at late $\mathrm{P} 0$. This axon had already formed a branch at the beginning of the video session (arrowhead in $A$ ). Three more branches formed de novo over the next $2 \mathrm{hr}$ (arrowheads in $B, D, E$ ). Two of these branches were directed ventrally toward the basilar pons $(B, D)$, whereas one $(E)$ extended dorsally. Sagittal section; dorsal is to the top, rostral to the left. Time is given in $\mathrm{hr}$ and min. These images were embossed (see Materials and Methods).

The dynamic behaviors observed along the axon shaft overlying the basilar pons were not evident at positions just caudal to the basilar pons, where collateral projections are not established. This spatial restriction in dynamic axon behavior could be attributable either to positive cues in the region of the basilar pons that induce branching or to negative cues in certain areas of the tract that inhibit branching. Molecules that inhibit or repel growth cones have been implicated in defining axonal growth trajectories during the development of several axonal projections (for review, see Keynes and Cook, 1995). One such molecule, a repulsive activity associated with chick caudal tectal membranes originally defined by its ability to repel and collapse the growth cones of retinal axons in vitro (Walter et al., 1987a,b; Stahl et al., 1990), can also inhibit the branching of retinal axons in vitro (Roskies and O'Leary, 1994). It is possible, therefore, that inhibitory molecules localized to parts of the corticospinal tract inhibit branching activity. Other studies, however, indicate a pivotal role for the basilar pons itself in regulating branching (O'Leary et al., 1991). Collagen gel coculture experiments have shown that pontine tissue releases a chemotropic activity that attracts cortical axons (Heffner et al., 1990). Similar in vitro experiments using time-lapse video microscopy have indicated that the pontine-derived chemoattractant also enhances the formation of branches from the shaft of cortical axons (Sato et al., 1994).

In addition to a spatial restriction in activity along the axon shaft, we find that the observed dynamic phenomena are also temporally restricted, indicating a critical period for axonal branching. This critical period could be attributable to changes in axonal responsiveness, to changes in the distribution of cues that induce or inhibit branching, or to a combination of these factors. Other components that do not directly induce or inhibit branch formation nonetheless may alter axonal responsiveness to the cues that do. For example, in the spinal cord where corticospinal axons also innervate the spinal gray via the mechanism of interstitial collateral branching, polysialic acid (PSA), a sugar epitope on the neural cell adhesion molecule, is selectively upregulated on corticospinal axons coincident with the onset of collateral branching. The removal of PSA both delays and diminishes branch formation (M. Daston, M. Bastmeyer, U. Rutishauser, D. O'Leary, unpublished data).

We observed that $77 \%$ of the branches that formed de novo were extended ventrally from the axon shaft directly toward the basilar pons. This directed extension was apparent from the onset of branch extension: we never observed a branch to extend dorsally and then redirect its growth ventrally toward the basilar pons; nor did we observe an "active site" on an axon shaft that extended a branch dorsally and later extended a branch ventrally. Indeed, of the countless thousands of branches that we have labeled and examined in tissue sections of fixed brains using conventional fluorescence microscopy (as in O'Leary and Terashima, 1988), we have seen only a single example of a collateral branch that initially extended dorsally, but turned to redirect its trajectory ventrally toward the basilar pons (M. Bastmeyer and D. O'Leary, unpublished observations). Even the $20 \%$ of the branches that we 
Table 1. Quantitation of de novo interstitial branching: frequency, directionality, stabilization

\begin{tabular}{|c|c|c|c|c|c|}
\hline Axon number & $\begin{array}{l}\text { Number of } \\
\text { branches }\end{array}$ & $\begin{array}{l}\text { Direction of } \\
\text { branches }\end{array}$ & $\begin{array}{l}\text { Observation time } \\
(\mathrm{min})\end{array}$ & $\begin{array}{l}\text { Axon length } \\
\text { observation }(\mu \mathrm{m})\end{array}$ & Branches $\cdot 100 \mu \mathrm{m}^{-1} \cdot \mathrm{hr}^{-1}$ \\
\hline 1 & $2^{a}$ & $v, v$ & 160 & 200 & 0.38 \\
\hline 2 & $1^{a}, 2$ & $d, v, d$ & 170 & 300 & 0.35 \\
\hline 3 & $2^{a}$ & $v, v$ & 200 & 200 & 0.3 \\
\hline 4 & $2^{a}$ & $\mathrm{~d}, \mathrm{v}$ & 300 & 200 & 0.2 \\
\hline 5 & 1 & $\mathrm{v}$ & 170 & 200 & 0.18 \\
\hline 6 & 1 & $\mathrm{v}$ & 180 & 200 & 0.17 \\
\hline 7 & 1 & $\mathrm{v}$ & 200 & 200 & 0.15 \\
\hline 8 & $1^{a}$ & $\mathrm{v}$ & 300 & 200 & 0.1 \\
\hline 9 & 1 & $\mathrm{v}$ & 300 & 200 & 0.1 \\
\hline 10 & 1 & $\mathrm{v}$ & 320 & 200 & 0.09 \\
\hline 11 & 1 & $\mathrm{v}$ & 320 & 200 & 0.09 \\
\hline 12 & $1^{a}$ & $\mathrm{v}$ & 320 & 200 & 0.09 \\
\hline 13 & $1^{a}$ & d & 320 & 200 & 0.09 \\
\hline 14 & 0 & & 300 & 200 & 0 \\
\hline 15 & 0 & & 300 & 200 & 0 \\
\hline Totals & $18 / 10^{a}$ & $14 \mathrm{v}, 4 \mathrm{~d}$ & 3860 & 3100 & \\
\hline Mean/axon & 1.2 & & 257 & 206 & $0.15 \pm 0.03$ \\
\hline
\end{tabular}

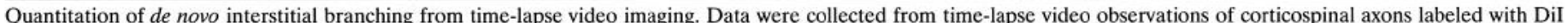

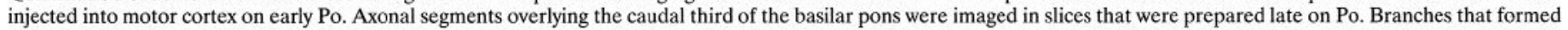

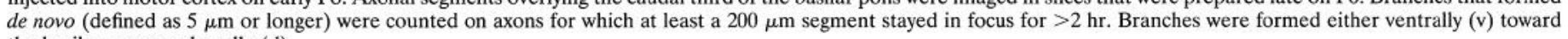
the basilar pons or dorsally (d).

${ }^{a}$ Branches that still persisted at the end of the recordings.
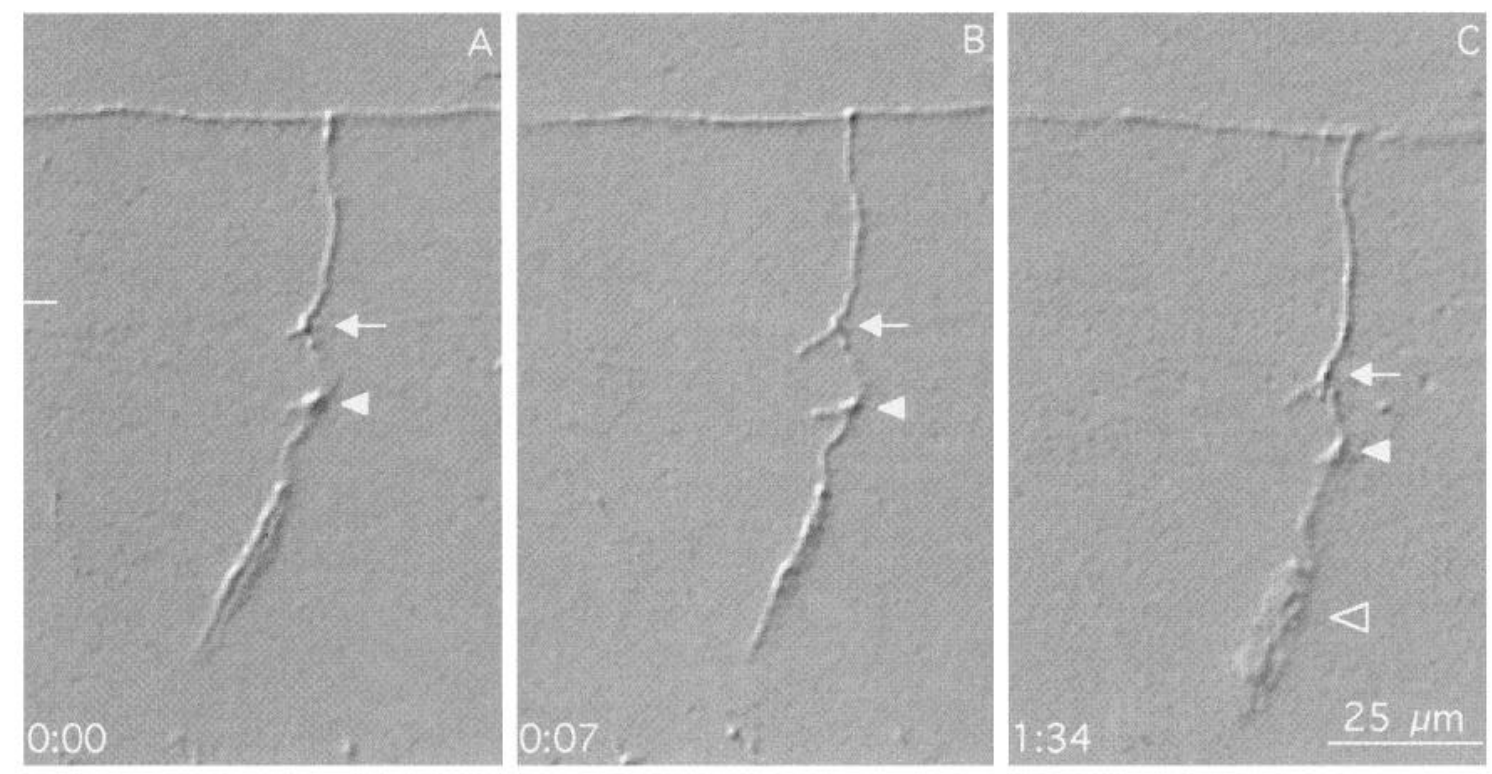

Figure 7. Collateral branch formation is temporally restricted. Corticospinal axon labeled with DiI injected into motor cortex and imaged at late P1. By late P1, long collateral branches have extended into the basilar pons (the border between the axon tract and the pontine neuropil is marked by a white line in $A$ ). Neither branch formation nor axonal activity was observed along corticospinal axons at this later age; however, the collateral branches themselves show considerable activity within the pontine neuropil. Two secondary branches are marked by an arrow and a filled arrowhead. One branch elongated and remained stable over the next 90 min (arrows in $A-C$ ), whereas the other branch elongated and retracted again (filled arrowhead in $A-C$ ). During the observation time, the ending of the main branch elaborated a complex arbor within the pontine neuropil (open arrowhead in $C$ ). Sagittal section; dorsal is to the top, rostral to the left. Time is given in hr and min. These images were embossed (see Materials and Methods).

observed with time-lapse video imaging to extend dorsally do not necessarily represent misdirected branches because structures dorsal to the tract, like the nucleus reticularis tegmenti pontis (Torigoe et al., 1986), also receive a collateral projection from corticospinal axons. A directional action of a pons-derived chemoattractant has been observed with time-lapse video imaging of cortical axons cocultured with basilar pons in collagen gels (Sato et al., 1994). Pontine explants enhanced by fivefold the formation of branches along cortical axons, and $\sim 70 \%$ of these branches were directed toward the pontine explant. These in vitro findings are consistent with the notion that a chemoattractant released by the basilar pons induces and directs the ingrowth of cortical axon 
branches. However, in contrast to our study, most of the activity was detected in an axon segment within $100 \mu \mathrm{m}$ of the growth cone. Furthermore, Sato et al. (1994) observed a high frequency of de novo branching $\left(\sim 1.5\right.$ branches $\left.\cdot \mathrm{hr}^{-1} \cdot \operatorname{axon}^{-1}\right)$ and a low stabilization rate $(2-3 \%)$. in comparison, we observed in the slice preparation a low frequency of de novo branch extension $(0.15$ branches $\cdot 100 \mu \mathrm{m}$ axon $^{-1} \cdot \mathrm{hr}^{-1}$ ) and a high stabilization rate $(55 \%)$. These differences between the in vivo and in vitro findings could be explained in two ways. First, neurites from cortical explants are reextending axons that have been severed during explantation, a process that might alter the distribution of receptors for the pons-derived activity, as well as other components that may participate in branch formation. Second, because the in vivo and in vitro environments are very different, it is likely that the interactions experienced by cortical axons are also very different. For example, in vivo cortical axons might have interactions that dampen branch formation, such as fasciculation within the tract, contact with molecules that inhibit branching, or other interactions that might help promote branch stabilization, such as contact with other cellular structures or various adhesion or extracellular matrix molecules.

The importance of interstitial axon branching in establishing axonal connections during vertebrate neural development is becoming increasingly apparent. In addition to its role in the for mation of cortical layer 5 projections, this mechanism appears to be responsible for the development of the axonal projections from the hippocampal formation to the mamillary bodies (Stanfield et al., 1987; Stanfield and O'Leary, 1988), the dorsal root ganglion neurons to the spinal gray (Snider et al., 1992), cortical callosal connections (Halloran and Kalil, 1994), and the innervation of the dorsal lateral geniculate nucleus (Bhide and Frost, 1991) and the optic tectum/superior colliculus (Nakamura and O'Leary, 1989; Simon and O'Leary, 1992a,b) by retinal axons. In addition, timelapse video microscopy of developing retinotectal axons in Xenopus (Harris et al., 1987; O'Rourke et al., 1994) and zebrafish (Kaethner and Stuermer, 1992) has revealed a related behavior, termed back-branching, characterized by the formation of several short terminal branches in close proximity to the growth cone or along branches of the developing arbor itself as a mechanism of arbor elaboration within the target.

In conclusion, our findings demonstrate that the axon shaft is responsible for the process of target recognition by cortical layer 5 axons. Although the primary growth cone of these axons does not seem to be involved in target recognition, it does serve the important roles of making specific pathway choices and navigating the axon along a subcortical pathway that places the axon shaft in proximity to its targets. Locally acting cues later induce the axon shaft to initiate and direct the extension of collateral branches into its targets. Regardless of the nature of these cues that promote target recognition, the axon shaft and the branches that it generates respond in a precise and specific manner.

\section{REFERENCES}

Bhide PG, Frost DO (1991) Stages of growth of hamster retinofugal axons: implication for developing axonal pathways with multiple targets. J Neurosci 11:485-504.

Bray D, Thomas C, Shaw G (1978) Growth cone formation in cultures of sensory neuron. Proc Natl Acad Sci USA 75:5226-5229.

de Kort EJM, Gribnau AAM, Van Aanholt HTH, Nieuwenhuys R (1985) On the development of the pyramidal tract in the rat. I. The morphology of the growth zone. Anat Embryol 172:195-204.

Ghosh A, Shatz CJ (1992) Pathfinding and target selection by developing geniculocortical axons. J Neurosci 12:39-55.
Godement P, Wang L-C, Mason CA (1994) Retinal axon divergence in the optic chiasm: dynamics of growth cone behavior at the midline. J Neurosci 14:7024-7039.

Halloran MC, Kalil K (1994) Dynamic behaviors of growth cones extending in the corpus callosum of living cortical brain slices observed with video microscopy. J Neurosci 14:2161-2177.

Harris WA, Holt CE, Bonhoeffer F (1987) Retinal axons with and without their somata, growing to and arborizing in the tectum of Xenopus embryos: a time-lapse video study of single fibres in vivo. Development 101:123-133.

Heffner CD, Lumsden AGS, O'Leary DDM (1990) Target control of collateral extension and directional axon growth in the mammalian brain. Science 247:217-220.

Honig MG, Hume RI (1989) DiI and DiO: versatile fluorescent dyes for neuronal labeling and pathway tracing. Trends Neurosei 12:333-341.

Joshi HC, Baas P, Chu DT, Heidemann SR (1986) The cytoskeleton of neurites after microtubule depolymerization. Exp Cell Res 163:233-245.

Kadhim HJ, Bhide PG, Frost DO (1993) Transient axonal branching in the developing corpus callosum. Cerebral Cortex 3:551-566.

Kaethner RJ, Stuermer CAO (1992) Dynamics of terminal arbor formation and target approach of retinotectal axons in living zebrafish embryos: a time-lapse study of single axons. J Neurosci 12:3257-3271.

Keynes RJ, Cook MW (1995) Repulsive and inhibitory signals. Curr Opin Neurobiol 5:75-82.

Kuang RZ, Kalil K (1994) Development of specificity in corticospinal connections by collaterals branching selectively into appropriate spinal targets. J Comp Neurol 344:270-282.

Mitchison T, Kirschner M (1988) Cytoskeletal dynamics and nerve growth. Neuron 1:761-772.

Myers PZ, Bastiani MJ (1993) Growth cone dynamics during the migration of an identified commissural growth cone. J Neurosci 13:127-143.

Nakamura H, O'Leary DDM (1989) Inaccuracies in initial growth and arborization of chick retinotectal axons followed by course corrections and axon remodeling to develop topographic order. J Neurosci 9:3776-3795

O'Connor TP, Duerr JS, Bentley D (1990) Pioneer growth cone steering decisions mediated by single filopodial contacts in situ. J Neurosci 10:3935-3946.

O'Leary DDM, Stanfield BB (1985) Occipital cortical neurons with transient pyramidal tract axons extend and maintain collaterals to subcortical but not intracortical targets. Brain Res 336:326-333.

O'Leary DDM, Terashima T (1988) Cortical axons branch to multiple subcortical targets by interstitial axon budding: implications for target recognition and "waiting periods." Neuron 1:901-910.

O'Leary DDM, Bicknese AR, De Carlos JA, Heffner CD, Koester SE, Kutka LJ, Tcrashima T (1990) Target selection by cortical axons: alternative mechanisms to establish axonal connections in the developing brain. In: Cold Spring Harbor symposia on quantitative biology, Vol LV, pp 453-367. Cold Spring Harbor, NY: Cold Spring Harbor Laboratory.

O'Leary DDM, Heffner CD, Kutka L, Lopez-Mascaraque L, Missias A, Reinoso BS (1991) A target-derived chemoattractant controls the development of the corticopontine projection by a novel mechanism of axon targeting. Development [Suppl] 2:123-130.

O'Rourke NA, Fraser SE (1990) Dynamic changes in optic fiber terminal arbors lead to retinotopic map formation: an in vivo confocal microscopic study. Neuron 5:159-171.

O'Rourke NA, Cline HT, Fraser SE (1994) Rapid remodeling of retinal arbors in the tectum with and without blockade of synaptic transmission. Neuron 12:921-934.

Roskies AL, O'Leary DDM (1994) Control of topographic retinal axon branching by inhibitory membrane-bound molecules. Science 265:799-803.

Sabry IH, O'Connor TP, Fvans I, Toroian-Raymond A, Kirschner M, Bentley D (1991) Microtubule behavior during guidance of pioneer neuron growth cones in situ. J Cell Biol 115:381-395.

Sato M, Lopcz-Mascaraque L, I Icffncr CD, O'Lcary DDM (1994) Action of a diffusible target-derived chemoattractant on cortical axon branch induction and directed growth. Neuron 13:791-803.

Simon DK, O'Leary DDM (1992a) Development of topographic order in the mammalian retinocollicular projection. J Neurosci 12:12-1232.

Simon DK, O'Leary DDM (1992b) Responses of retinal axons in vivo and in vitro to position-encoding molecules in the embryonic superior colliculus. Neuron 9:1-20. 
Snider WD, Zhang L, Yusoof S, Gorukani N, Tsering C (1992) Interaction between dorsal root axons and their target motor neurons in developing mammalian spinal cord. J Neurosci 12:3494-3508.

Sretavan DW, Reichardt LF (1993) Time-lapse video analysis of retinal ganglion cell axon pathfinding at the mammalian optic chiasm: growth cone guidance using intrinsic chiasm cues. Neuron 10:761-777.

Stahl B, Müller B, Boxberg Y, von Cox EC, Bonhoeffer F (1990) Biochemical characterization of a putative axonal guidance molecule of the chick visual system. Neuron 5:735-743.

Stanfield BB, O'Leary DDM (1988) Neurons in the subiculum with transient postmammillary collaterals during development maintain projections to the mammillary complex. Exp Brain Kes 72:185-190.

Stanfield BB, Nahin BR, O'Leary DDM (1987) A transient postmamillary component of the rat fornix during development: implications for interspecific differences in mature axonal projections. $\mathrm{J}$ Neurosci 7:3350-3361.

Torigoe Y, Blanks RHI, Precht W (1986) Anatomical studies on the nucleus reticularis tegmenti pontis in the rat. I. Cytoarchitecture, topography, and cerebral cortical afferents. J Comp Neurol 243:71-87

Walter J, Kern-Veits B, Huf J, Stolze B, Bonhoeffer F (1987a) Recognition of position-specific properties of tectal cell membranes by retinal axons in vitro. Development 101:685-696.

Walter J, Henke-Hahle S, Bonhoeffer $\mathbf{F}$ (1987b) Avoidance of posterior tectal membranes by temporal retinal axons. Development 101:909-913.

Yu W, Ahmad FJ, Baas PW (1994) Microtubule fragmentation and partitioning in the axon during collateral branch formation. J Neurosci $14: 5872-5884$. 